\title{
Direct Evidence of Shelled Structures of Au-Pd Bimetallic Nano-Particles
}

\author{
J. P. Zhang*, D. T. Foord***, J. Chou**, and E. W. McFarland** \\ *Materials Dept., ** Chemical Engineering Dept., Univ. of California, Santa Barbara, CA 93106; \\ ***FEI Company, 5350 NE Dawson Creek Drive., Hillsboro, OR 97124
}

Bimetallic surfaces and alloys have demonstrated very interesting catalytical properties for chemical transformations. Recently a micelle encapsulation based on block copolymers has been used to synthesize metal nanoparticles such as $\mathrm{Au}$ and $\mathrm{Pd}$ previously with great success to produce metal nanoparticles with uniform size distribution [1] and we have developed this routine to synthesize Au with $14 \%$ wt. $\mathrm{Pd}$ nanoclusters in cores of micelles by mixing $\mathrm{Au}^{3+}$ and $\mathrm{Pd}^{2+}$ cations. After removing the surrounding polymer in calcinations, the Au-Pd nanoparticles, in size of 5 to $10 \mathrm{~nm}$ supported on $\mathrm{TiO}_{2}$, exhibit significantly improved activity for propylene coupling to benzene. In this work we present the microscopic studies of these particles. A shelled structure of bimetallic particles has been observed that the gold particles are commonly covered by thin palladium layers in the size range we studied.

Au-Pd particles dispersed on clean carbon film were examined under a FEI Tecnai TF30UT (300kV HT, FEG source with an ultra twin lens) for atomic imaging and elemental line scan with beam sizes under 1 nanometer, and a JEOL2010 (200 kV LaB $)$ machine for statistical EDX (electronon dispersive X-ray) measurements of larger particles. The line scans, as an example shown in Fig.1, indicated palladium only located on the surface area, and the palladium profile showed two sharp peaks, while the gold content dropped significantly, can be interpreted as a shelled structure and the thickness of that shell was about $4.6 \mathrm{~nm}$ there. Pd surface domains were also observed in atomic images, as shown in Fig.2.

It is also verified with a statistics of twenty particles that, when the size is getting smaller, the gold content in core is reducing: about $6 \%$ of palladium when the diameter is $30 \mathrm{~nm}$, and, when below 5 $\mathrm{nm}$, the particles are mainly of palladium. The survey also shows there is a preferred size range of 8 to $10 \mathrm{~nm}$ for Au-Pd bimetallic particles, where the particles can be distributed near homogeneously. Interestingly, samples in this range have been found to behave the best yield of benzene formation.

Several attempts have been made to elucidate the formation of Au-Pd bimetallic phase [2], which is in contrast to the fact that no gold-palladium compound has been found in bulk material. Our work may help to understand this phenomena that the bimetallic Au-Pd phase could be formed in a nanostructure, where the palladium can only stay on the surface of the gold core, and, when the particle size varies, its mainly due to the change of gold in core, rather than palladium on surface. Therefore only surface alloy of Au-Pd is possible.

\section{References}

[1] J. Chou, et al, Catal. Lett., 95 (2004)107. J. Chou, et al, Angew. Chem. Int. Ed., In revision.

[2] L. Guczi, et al, J. Mol. Catal. A, Che. 204-205 (2003) 545; C.J. Baddeley, et al., J. Phys. Chem., 99 (1995) 5146; H. Liu, et al., J. Mol. Catal., 74 (1992) 275.

[3] This work made use of MRL Central Facilities supported by the MRSEC Program of the National Science Foundation under award No. DMR00-80034. 
(a)
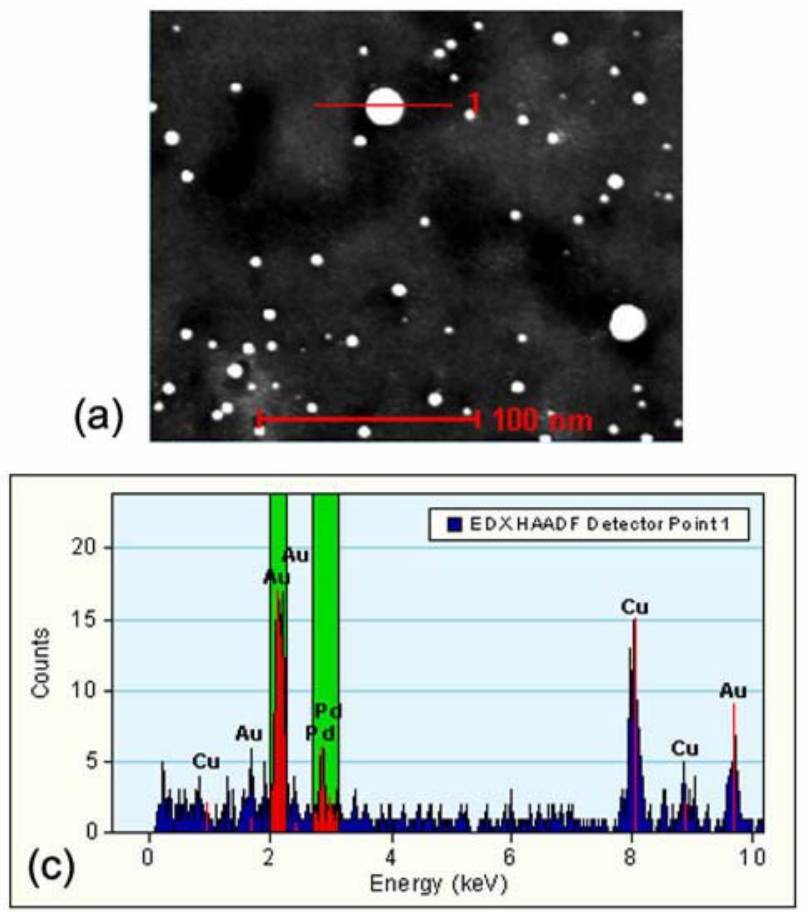
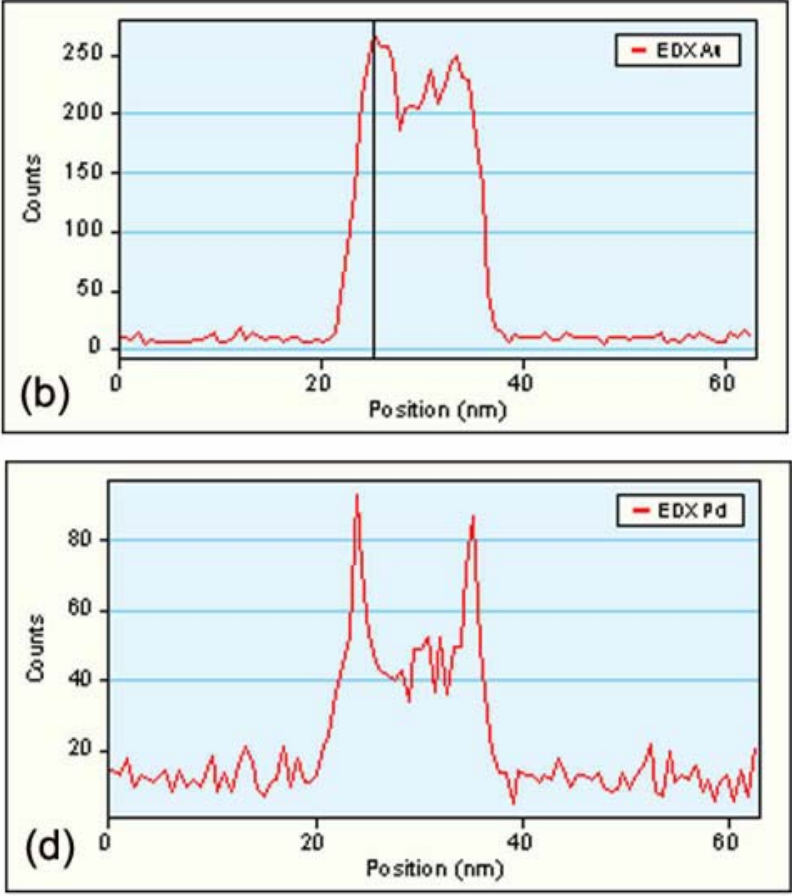

FIG. 1. Line scan of a $17 \mathrm{~nm}$ AuPd particle, shown in (a), with selected EDX widows of Au -M and Pd-L in (c). The corresponding profiles in (b) and (d) indicate the palladium located on surface of the gold particle in a shell of about $4.6 \mathrm{~nm}$ thick. The black line in (b) marks the interface of the Pd-shell with the gold core, the corresponding spectrum at that position is shown in (c).

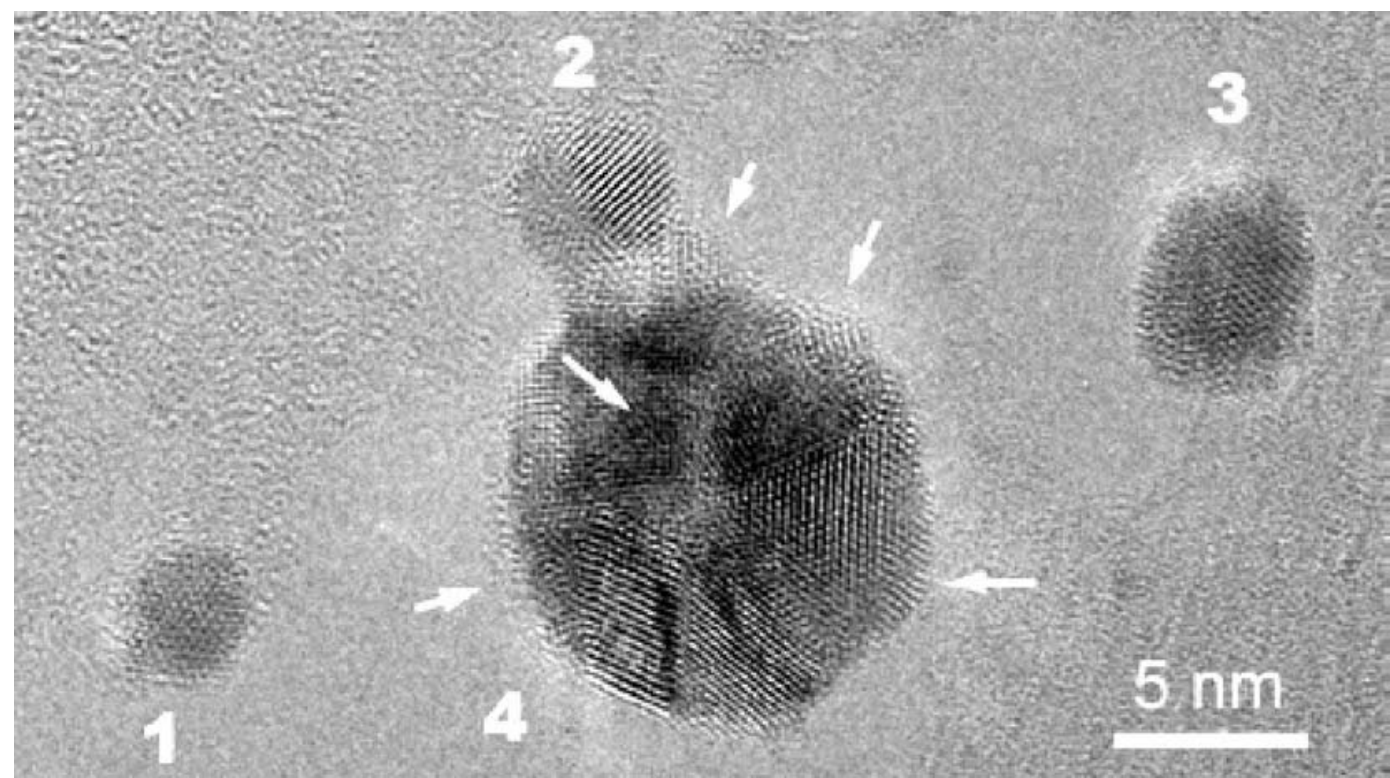

FIG. 2 With the results from X-ray analysis shown above, the surface domains (arrowed) on a fivefold gold twins (No.4), are identified as palladium. The smaller particles (labeled as 1,2,3), of which the diameter is less than $5 \mathrm{~nm}$, are mainly of single phased. 